Research Square
Preprints are preliminary reports that have not undergone peer review.

They should not be considered conclusive, used to inform clinical practice, or referenced by the media as validated information.

\title{
Changes in physical activity across pregnancy among Chinese women: a longitudinal cohort study
}

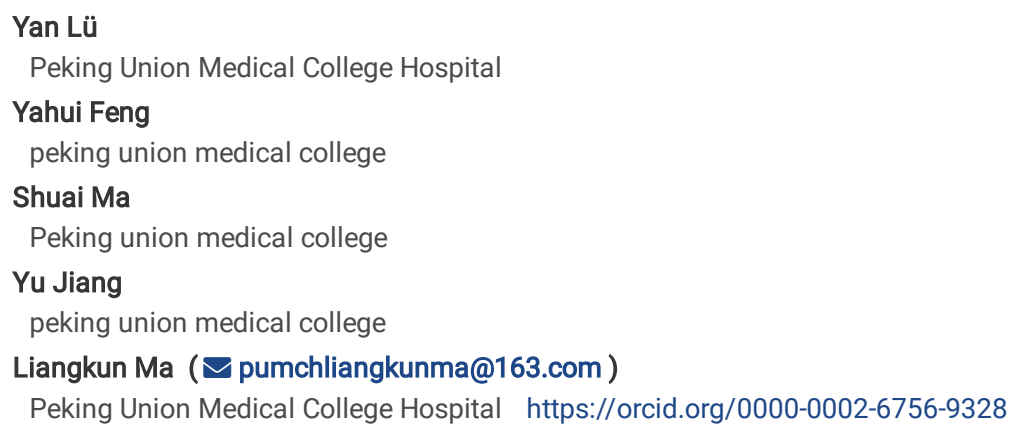




\section{Abstract}

Background Sufficient physical activity (PA) during pregnancy is beneficial for a woman's health; however, the PA levels of Chinese women at different pregnancy stages are not clear. The aim of our study was to investigate PA changes during pregnancy and determinants of PA changes among Chinese women.

Methods Data were obtained from 2485 participants who were enrolled in the multicentre prospective Chinese Pregnant Women Cohort Study. PA level was assessed in early pregnancy and again in mid-to-late pregnancy using the International Physical Activity Questionnaire short form (IPAQ-SF). Sufficient PA ( $\geq 600 \mathrm{MET}$ min/week) in early pregnancy and insufficient PA in mid-to-late pregnancy indicated decreasing PA. Insufficient PA in early pregnancy and sufficient PA in mid-to-late pregnancy indicated increasing PA. The associations between demographic, pregnancy-related and health-related characteristics and PA changes were examined by logistic regression analysis.

Results Energy expenditure on PA increased significantly from early to mid-to-late pregnancy $(P<0.001)$, and $55.25 \%$ of the participants eventually had sufficient PA. Walking was the dominant form of PA. Women with sufficient PA levels in early pregnancy were more likely to have sufficient PA in mid-to-late pregnancy ( $\mathrm{OR}=1.897,95 \% \mathrm{Cl}$ : 1.583-2.274; $\mathrm{P}<0.001)$. Women in West China and those in Central China were most and least likely, respectively, to have increasing PA ( $O R=1.387,95 \% \mathrm{Cl}: 1.078-1.783 ; \mathrm{P}=0.011$ vs. $\mathrm{OR}=0.721,95 \% \mathrm{Cl}$ : 0.562-0.925; $\mathrm{P}=0.010)$. Smoking was inversely associated with increasing $\mathrm{PA}$ $(\mathrm{OR}=0.480,95 \% \mathrm{Cl}: 0.242-0.955 ; \mathrm{P}=0.036)$. Women with higher educational levels were less likely to have decreasing $\mathrm{PA}(\mathrm{OR}=0.662,95 \% \mathrm{Cl}: 0.442-0.991 ; \mathrm{P}$ $=0.045)$.

Conclusions PA increased as pregnancy progressed, and walking was the dominant form of PA among Chinese women. Avoiding smoking and maintaining sufficient PA in early pregnancy may help women achieve sufficient PA throughout the whole pregnancy. Interventions for PA should consider differences in regional distributions and educational levels.

\section{Background}

Physical activity (PA) is bodily movement that is carried out by skeletal muscles, and PA results in energy expenditure in the domains of occupation, the household, transportation, leisure and exercise [1]. PA during pregnancy is not only safe [1,2] but also helpful in improving physical health, controlling weight, alleviating back pain, accelerating postpartum recovery and reducing the risk of gestational diabetes, preeclampsia and operative delivery [2-14]. To achieve good health, the World Health Organization (WHO) recommends that adults engage in at least 150 minutes of moderate-intensity PA throughout the week or an equivalent form of PA that achieves at least 600 metabolic equivalent task (MET) min/week $[1,15]$. The department of Health and Human Services of the United States recommends that pregnant women engage in the same level of PA as that recommended by the WHO for adults [16].

Although the benefits of PA have been well documented, PA decreases during pregnancy compared with the prepregnancy period [17, 18]. The proportion of women achieving the recommended PA level varies widely from 11-66\% worldwide, and this wide range is mainly due to different population characteristics and assessment times [18-20]. It is crucial to clarify the PA level at different pregnancy stages before implementing effective interventions; however, although previous studies have compared PA levels before and during pregnancy, few have investigated changes in PA over the course of pregnancy.

Chinese families attach high importance to pregnant women and their foetuses and adhere to the traditional philosophy of protecting the foetus from miscarriage by advising pregnant women to rest as much as possible [21]. This idea sets up many obstacles to PA during pregnancy; however, young women are accepting of modern healthcare-based recommendations and actively engage in PA. Even so, the current level of PA during pregnancy among Chinese women is not clear. Previous studies are limited by their cross-sectional natures and represent only one city or district of China [20,22].

Therefore, our study aims to investigate PA changes between early and mid-to-late pregnancy and the determinants associated with PA changes among Chinese women. Data were obtained from the Chinese Pregnant Women Cohort Study (CPWCS). The CPWCS is a multicentre prospective observational study designed to investigate the lifestyles of pregnant Chinese women and the associations between lifestyle and obstetric or neonatal outcomes.

\section{Methods}

\section{Study design and population}

The CPWCS recruited pregnant women from 14 maternal and child healthcare hospitals and 10 academic hospitals located in 15 provinces of China. Seven, nine and eight hospitals were in East, Central, and West China, respectively. Each hospital has approximately 400 deliveries per month. Pregnant women who received early pregnancy evaluations at each study recruitment hospital within a certain month from July 2017 to November 2018 were screened for eligibility. The inclusion criteria were as follows: women were aged 16 years or above, with a gestational age of 5-12 weeks; were permanent residents of the study recruitment district; had the intention of delivering in the study recruitment hospital; and had agreed to participate in the CPWCS. The exclusion criteria were women with serious chronic diseases and psychosis. The CPWCS was approved by the ethics review committee of Peking Union Medical College Hospital, the leading centre (HS-1345). Written informed consent was obtained from all participants.

Among all participants in the CPWCS, only those who could recall their PA over the previous 7 days when being surveyed were eligible for our PA study. Women were excluded from the PA study if they had a multiple pregnancy $(n=32)$, a miscarriage $(n=74)$, or a chronic disease ( $n=102)$ including hypertension, diabetes, heart disease, renal disease, or other diseases that would restrict PA during pregnancy. A total of 2485 women with PA information in both early and mid-to-late pregnancy were finally included for analysis in our study. 
Demographic, pregnancy-related and health-related data were obtained at the initial recruitment clinic visit in early pregnancy (mean=10.7 weeks of gestation). The PA level was assessed at the initial recruitment clinic visit and updated at the prenatal clinic visit in mid-to-late pregnancy (mean=32.0 weeks of gestation).

\section{Population characteristics}

Information on age, residential region, ethnicity, educational level, annual household income, occupation, parity, pregnancy plan, height, prepregnancy weight, and history of smoking or drinking was obtained at the initial recruitment clinical visit. Residential regions were categorized into East, Central and West China according to the Chinese Health Statistics Yearbook. The prepregnancy body mass index (BMI) $\left(\mathrm{kg} / \mathrm{m}^{2}\right)$ was calculated based on the self-reported prepregnancy weight in kilograms and height in centimetres. BMI was categorized as underweight, normal weight, overweight and obese (<18.5, 18.5-23.9, $\geq 24$, respectively) $[23,24]$.

\section{Assessment of PA}

PA was assessed in early and mid-to-late pregnancy using the International Physical Activity Questionnaire short form (IPAQ-SF) validated for the Chinese population $[25,26]$. The IPAQ addresses three types of PA: high-intensity PA, medium-intensity PA, and walking. High-intensity PA refers to activities that require hard physical effort and that make breathing much harder than normal, such as heavy lifting, digging, or aerobics [27]. Medium-intensity PA refers to activities that take moderate physical effort and make breathing somewhat harder than normal, such as carrying light loads, bicycling at a regular pace, or table tennis [27]. Walking includes all walking for occupation, transportation, household, exercise and leisure. The frequency (days) and duration (minutes) of each PA over the previous seven days were investigated. Total energy expenditure (TEE) on PA per week was calculated as a total of three types of PA reported in the MET value $\times$ minutes per week. The values of 3.3, 4.0 and 8.0 were assigned to represent the MET values of walking, medium-intensity PA and highintensity PA, respectively [28].

According to the IPAQ-SF, TEE on PA $\geq 600$ MET min/week is defined as "moderate level" [28], and WHO recommends a minimum of 600 MET min/week PA to realize a health benefit [15]. Therefore, we defined PA with TEE $\geq 600 \mathrm{MET}$ min/week as sufficient PA and PA with TEE $<600 \mathrm{MET}$ min/week as insufficient PA. Sufficient PA in early pregnancy and insufficient PA in mid-to-late pregnancy indicated decreasing PA. Insufficient PA in early pregnancy and sufficient PA in mid-to-late pregnancy indicated increasing PA.

\section{Statistical analysis}

The population characteristics of all women included in the study are described. Categorical data are expressed as frequencies and percentages. Continuous data are expressed as means, standard deviations (SDs), medians and interquartile ranges (IQRs). TEE on PA, energy expenditure on each type of PA, and the proportion of energy expenditure on each type of PA to TEE on PA were compared between early and mid-to-late pregnancy using the Wilcoxon signed-rank test. The proportions of women with sufficient PA levels were compared between early and mid-to-late pregnancy using McNemar's test. Univariate and multivariate logistic regression were used to calculate the unadjusted and adjusted odds ratios (ORs) and $95 \%$ confidence intervals (Cls) to address the following: (1) associations between population characteristics and sufficient PA in mid-to-late pregnancy among all women included in the study, (2) associations between population characteristics and increasing PA among the subset of women with insufficient PA levels in early pregnancy, and (3) associations between population characteristics and decreasing PA among the subset of women with sufficient PA levels in early pregnancy. $P$ values $<0.05$ were considered statistically significant. SPSS 22.0 (IBM, Armonk, NY, USA) was used for statistical analysis.

\section{Results}

\section{Population characteristics}

The characteristics of all participants are shown in Table 1. The median age was 28 years $(26,31)$. The proportions of women in East, Central and West China were similar. The vast majority of women (94.37\%) were of the Han ethnicity. Most women $(71.83 \%)$ had an educational level of college or above. The distribution of women at different household income levels was nearly equivalent. Most women (76.18\%) were employed, with $55.35 \%$ having a non-manual occupation and $20.85 \%$ having a manual occupation. More than half of the women were nulliparous (61.05\%). Most pregnancies (74.41\%) were planned. More than half of the women had normal weight (64.19\%). The vast majority of women had no history of smoking (97.55\%) or drinking (94.81\%).

\section{Change in PA across pregnancy}

Table 2 shows that TEE on PA and energy expenditure on each type of PA increased significantly from early to mid-to-late pregnancy $(P<0.001)$. The largest proportion of PA across pregnancy comprised walking (median, 100\%). The proportion of women with sufficient PA levels also increased significantly from $32.72 \%$ to $55.25 \%$ from early to mid-to-late pregnancy $(P<0.001)$. Among 1672 women with insufficient PA levels in early pregnancy, $836(50.00 \%)$ remained insufficient, and $836(50.00 \%)$ had increasing PA. Among 813 women with sufficient PA levels in early pregnancy, 537 (66.05\%) maintained sufficient PA, and 276 (33.95\%) had decreasing PA (see Fig. 1).

\section{Determinants of PA}

Table 3 shows that women in West China ( $\mathrm{OR}=1.247,95 \% \mathrm{Cl}$ : 1.012-1.537; $P=0.038)$ were most likely and that women in Central China (OR=0.747, $95 \% \mathrm{Cl}$ : $0.609-0.916 ; P=0.005)$ were least likely to have sufficient PA in mid-to-late pregnancy. Women with a smoking history (OR=0.551, 95\% $\mathrm{Cl}$ : $0.315-0.964$; $P=0.037)$ were less likely, and women with sufficient PA levels in early pregnancy (OR=1.897, $95 \% \mathrm{Cl}: 1.583-2.274 ; P<0.001)$ were more likely to have sufficient $\mathrm{PA}$ in mid-to-late pregnancy. Table 4 shows that in the subset of women with insufficient PA levels in early pregnancy, women in West China (OR=1.387, $95 \%$ 
Cl: $1.078-1.783 ; p=0.011)$ were most likely, and women in Central China (OR=0.721, $95 \% \mathrm{Cl}: 0.562-0.925 ; P=0.010)$ were least likely to increase PA across their pregnancies. Women with a smoking history (OR=0.480,95\% $\mathrm{Cl}: 0.242-0.955 ; P=0.036)$ were less likely to increase $\mathrm{PA}$ across their pregnancies. In the subset of women with sufficient PA levels in early pregnancy, women with educational levels of college or above $(0 \mathrm{R}=0.662,95 \% \mathrm{Cl}$ : $0.442-0.991 ; P=0.045)$ were less likely to decrease PA across their pregnancies.

\section{Discussion}

To our knowledge, this is the first multicentre longitudinal cohort study to investigate changes in PA across pregnancy in a Chinese population. We found that PA levels increased from early to mid-to-late pregnancy and that more than half of the women eventually had sufficient PA as recommended. Walking was the dominant form of PA. Women with sufficient PA levels in early pregnancy were more likely to maintain or achieve sufficient PA across their pregnancies. PA levels varied in different regions of China, with women in the West being most likely and those in the Central being least likely to have sufficient and increasing PA. Habitual smoking was inversely associated with sufficient and increasing PA. Women with higher educational levels were less likely to decrease PA across the pregnancy.

Our study found that the proportion of pregnant women achieving the recommended PA level increased from $32.72 \%$ in early pregnancy to $55.25 \%$ in mid-tolate pregnancy. Studies investigating PA levels at different pregnancy stages are limited. In contrast to our study, studies of western populations showed that PA decreased or remained unchanged as pregnancy progressed $[29,30]$. Regarding studies with Chinese populations, the proportion of women achieving the recommended level remained at a low level of $11 \%$ across the pregnancies of urban women from Tianjin [20] and increased from $53.8 \%$ in the first trimester to $61.4 \%$ in the third trimester among women from Chengdu [22]; however, both of these studies were cross-sectional, and the changes in PA were concluded from different subsets of women. Our study was a multicentre study, and PA was surveyed in the same sample of women longitudinally at different pregnancy stages, resulting in a better representation of the Chinese population and in less bias.

Our findings were consistent with those of one previous study using the IPAQ-SF, namely, that PA with medium or higher intensity contributed less to TEE during pregnancy [18]. Our study found that walking was the dominant form of PA. In this study, walking included all walking, namely, walking related to occupation, transportation, the household, exercise and leisure. Walking is the form of moderate-intensity PA indicated by the WHO recommendation and the form of exercise recommended by the American College of Obstetricians and Gynaecologist (ACOG) during pregnancy [1, 2, 15]. Chinese culture holds tight to traditional concepts of not walking fast, not running and not jumping during pregnancy, but walking is not restricted [21]. Therefore, it may be more reasonable and easier for prenatal healthcare providers to encourage women to walk appropriately to meet the sufficient level of $600 \mathrm{MET}$ min/week than to persuade them to participate in other forms of PA.

It has been well documented that prepregnancy PA habits are strongly associated with PA levels during pregnancy [29, 30]. Our study found that women with sufficient PA levels at baseline were more likely to maintain or achieve sufficient PA across their pregnancies, further validating the fact that a good lifestyle is beneficial in the long term.

West China is the region that has the lowest socioeconomic level and the largest ethnic minority population in China, and the pregnant women there were most likely to have sufficient and increasing PA. However, the multivariate logistic regression controlled for the confounders of ethnicity, education, income and occupation, and the results still indicated that the region was associated with sufficient and increasing PA. Therefore, the association might be due to the region-specific lifestyle rather than just ethnicity and socioeconomic status. When implementing interventions for PA, the focus should be placed on Central China, followed by Eastern and Western China.

Among the other determinants investigated, a history of smoking was inversely associated with sufficient and increasing PA. The findings were consistent with other study data showing that smokers had a more sedentary lifestyle [31]. The combined risk of smoking and low PA levels may make women more vulnerable to adverse pregnancy outcomes.

A recent systemic review revealed that younger age, higher education, higher income, employment, nulliparity, and normal weight were positively associated with PA during pregnancy, but the correlations were weak [29]. However, our study found that except for educational level, these factors were not associated with changes in PA across pregnancy. In our study, women with higher education tended to maintain sufficient PA throughout the whole pregnancy.

There were several limitations in our study. First, the study might have bias due to the self-report measure used to assess PA. However, this subjective bias might affect only the absolute value of PA and had little influence on the relative change in PA across pregnancy, since PA was assessed by the same IPAQ-SF in both early and mid-to-late pregnancy. Second, the generalization of our results might be limited to convenience sampling and the recruitment of only participants who could recall their PA. Finally, our study did not include discomfort during pregnancy and subjective factors, such as self-efficacy or perceived behavioural control, which might influence PA [32,33]. Further analyses that include these factors as determinants of PA are needed.

\section{Conclusions}

To our knowledge, our study is the first multicentre longitudinal cohort study to investigate changes in PA across pregnancy among Chinese women. Our findings indicated that PA increased as pregnancy progressed and that walking was the dominant form of PA. Avoiding smoking and having sufficient PA in early pregnancy may help women achieve sufficient PA throughout the whole pregnancy, suggesting that pregnancy is the ideal time for maintaining or adopting a healthy lifestyle. Our findings also suggest that interventions for PA should consider differences in regional distributions and educational levels. The trend towards PA changes across pregnancy and the determinants associated with PA in our study may help to develop appropriate interventions for PA to improve maternal health among Chinese women. 


\section{Abbreviations}

ACOG: American College of Obstetricians and Gynecologists; BMI: Body mass index; Cl: Confidence interval; CPWCS: Chinese Pregnant Women Cohort Study; IPAQ-SF: International Physical Activity Questionnaire short form; IQR: Interquartile range; MET: Metabolic equivalent task; OR: Odds ratio; PA: Physical activity; SD: Standard deviation; TEE: Total energy expenditure; WHO: World Health Organization

\section{Declarations}

\section{Ethics approval and consent to participate}

The study was approved by the ethics review committee of Peking Union Medical College Hospital, the leading centre (HS-1345). Written informed consent was obtained from each participant.

\section{Consent for publication}

Not applicable

\section{Availability of data and materials}

The datasets used and/or analysed during the current study are available from the corresponding author on reasonable request.

\section{Competing interests}

The authors declare that they have no competing interests.

\section{Funding}

The study was supported by the Fundamental Research Funds for the Central Universities (Grant No. 3332018019). The funding body was not involved in the design of the study; in the collection, analysis, or interpretation of the data; or in writing of the manuscript.

\section{Authors' contributions}

YL, LM, and YJ conceptualized and designed the study; SM collected the data; YF performed the statistical analyses; and YL drafted the manuscript. All authors have approved the final version of the paper for publication.

\section{Acknowledgements}

The authors thank all of the study participants for their willingness to participate in this study. The authors thank AJE (http://www.aje.cn/) for the English language review.

\section{References}

1. World Health Organization. WHO guidelines approved by the guidelines review committee. Global recommendations on physical activity for health. Geneva: World Health Organization; 2010.

2. American College Of Obstetricians Gynecologists. Physical activity and exercise during pregnancy and the postpartum period. Committee Opinion. 2015;650:e135-42.

3. Price BB, Amini SB, Kappeler K. Exercise in pregnancy: effect on fitness and obstetric outcomes-a randomized trial. Med Sci Sports Exerc. $2012 ; 44: 2263-9$.

4. Kramer MS, McDonald SW. Aerobic exercise for women during pregnancy. Cochrane Database Syst Rev. 2006;CD000180; doi:10.1002/14651858.CD000180.pub2.

5. Haakstad LA, Voldner N, Henriksen T, Bo K. Physical activity level and weight gain in a cohort of pregnant Norwegian women. Acta Obstet Gynecol Scand. 2007;86:559-64.

6. Jiang H, Qian X, Li M, Lynn H, Fan Y, Jiang H, et al. Can physical activity reduce excessive gestational weight gain? Findings from a Chinese urban pregnant women cohort study. Int J Behav Nutr Phys Act. 2012;9:12.

7. Kihlstrand M, Stenman B, Nilsson S, Axelsson O. Water-gymnastics reduced the intensity of back/low back pain in pregnant women. Acta Obstet Gynecol Scand. 1999;78:180-5.

8. Dempsey JC, Sorensen TK, Williams MA, Lee IM, Miller RS, Dashow EE, et al. Prospective study of gestational diabetes mellitus risk in relation to maternal recreational physical activity before and during pregnancy. Am J Epidemiol. 2004;159:663-70.

9. Liu J, Laditka JN, Mayer-Davis EJ, Pate RR. Does physical activity during pregnancy reduce the risk of gestational diabetes among previously inactive women? Birth. 2008;35:188-95.

10. Saftlas AF, Logsden-Sackett N, Wang W, Woolson R, Bracken MB. Work, leisure-time physical activity, and risk of preeclampsia and gestational hypertension. Am J Epidemiol. 2004;160:758-65.

11. Aune D, Saugstad OD, Henriksen T, Tonstad S. Physical activity and the risk of preeclampsia: a systematic review and meta-analysis. Epidemiology. 2014;25:331-43. 
12. Domenjoz I, Kayser B, Boulvain M. Effect of physical activity during pregnancy on mode of delivery. Am J Obstet Gynecol. 2014;211:401.e1-11.

13. Ko YL, Chen CP, Lin PC. Physical activities during pregnancy and type of delivery in nulliparae. Eur J Sport Sci. 2016;16:374-80.

14. Barakat R, Pelaez M, Lopez C, Montejo R, Coteron J. Exercise during pregnancy reduces the rate of cesarean and instrumental deliveries: results of a randomized controlled trial. J Matern Fetal Neonatal Med. 2012;25:2372-6.

15. Global physical activity questionnaire (GPAQ) analysis guide. World Health Organization. 2013. https://www.who.int/ncds/surveillance/steps/resources/GPAQ_Analysis_Guide.pdf. Accessed 23 Feb 2020.

16. U.S. Health and Human Services Physical Activity Guidelines Advisory Committee. Chapter 7: additional considerations for some adults. In: Physical activity guidelines for Americans. U.S. Health and Human Services. 2008. http://www.health.gov/paguidelines/guidelines/chapter7.aspx. Accessed 23 Feb 2020 .

17. Fell DB, Joseph KS, Armson BA, Dodds L. The impact of pregnancy on physical activity level. Matern Child Health J. 2009;13:597-603.

18. Padmapriya N, Shen L, Soh SE, Shen Z, Kwek K, Godfrey KM, et al. Physical activity and sedentary behavior patterns before and during pregnancy in a multi-ethnic sample of Asian women in Singapore. Matern Child Health J. 2015;19:2523-35.

19. Pearce EE, Evenson KR, Downs DS, Steckler A. Strategies to promote physical activity during pregnancy: a systematic review of intervention evidence. Am J Lifestyle Med. 2013;7; doi:10.1177/1559827612446416.

20. Zhang Y, Dong S, Zuo J, Hu X, Zhang H, Zhao Y. Physical activity level of urban pregnant women in Tianjin, China: a cross-sectional study. PLoS One. 2014;9:e109624.

21. Lee DT, Ngai IS, Ng MM, Lok IH, Yip AS, Chung TK. Antenatal taboos among Chinese women in Hong Kong. Midwifery. 2009;25:104-13.

22. Xiang M, Zhang J, Liang H, Zhang Z, Konishi M, Hu H, et al. Physical activity and dietary intake among Chinese pregnant women: an observational study. BMC Pregnancy Childbirth. 2019;19:295.

23. Zhou B, Coorperative Meta-Analysis Group Of China Obesity Task Force. Predictive values of body mass index and waist circumference to risk factors of related diseases in Chinese adult population. Zhonghua Liu Xing Bing Xue Za Zhi. 2002;23:5-10.

24. Zhou BF, Cooperative Meta-Analysis Group of the Working Group on Obesity in China. Predictive values of body mass index and waist circumference for risk factors of certain related diseases in Chinese adults-study on optimal cut-off points of body mass index and waist circumference in Chinese adults. Biomed Environ Sci. 2002;15:83-96.

25. Craig CL, Marshall AL, Sjostrom M, Bauman AE, Booth ML, Ainsworth BE, et al. International physical activity questionnaire: 12-country reliability and validity. Med Sci Sports Exerc. 2003;35:1381-95.

26. Macfarlane DJ, Lee CC, Ho EY, Chan KL, Chan DT. Reliability and validity of the Chinese version of IPAQ (short, last 7 days). J Sci Med Sport. 2007;10:4551.

27. Deng HB, Macfarlane DJ, Thomas GN, Lao XQ, Jiang CQ, Cheng KK, et al. Reliability and validity of the IPAQ-Chinese: the Guangzhou biobank cohort study. Med Sci Sports Exerc. 2008;40:303-7.

28. Guidelines for data processing and analysis of the international physical activity questionnaire (IPAQ)-short and long forms. IPAQ Research Committee. 2005.

http://www.academia.edu/5346814/Guidelines_for_Data_Processing_and_Analysis_of_the_International_Physical_Activity_Questionnaire_IPAQ_Short_anc Accessed 23 Feb 2020.

29. Garland M, Wilbur J, Semanik P, Fogg L. Correlates of physical activity during pregnancy: a systematic review with implications for evidence-based practice. Worldviews Evid Based Nurs. 2019;16:310-8.

30. Gaston A, Cramp A. Exercise during pregnancy: a review of patterns and determinants. J Sci Med Sport. 2011;14:299-305.

31. Efendi V, Ozalevli S, Naz I, Kilinc O. The effects of smoking on body composition, pulmonary function, physical activity and health-related quality of life among healthy women. Tuberk Toraks. 2018;66:101-8.

32. Cramp AG, Bray SR. A prospective examination of exercise and barrier self-efficacy to engage in leisure-time physical activity during pregnancy. Ann Behav Med. 2009;37:325-34.

33. Downs DS, Devlin CA, Rhodes RE. The power of believing: salient belief predictors of exercise behavior in normal weight, overweight, and obese pregnant women. J Phys Act Health. 2015;12:1168-76.

\section{Tables}

Table 1. Demographic, pregnancy-related and health-related information of pregnant Chinese women $(\mathrm{n}=2485)$ 


\begin{tabular}{|c|c|c|}
\hline Characteristics & $\mathrm{N}$ & $\%$ \\
\hline \multicolumn{3}{|l|}{ Demographic characteristics } \\
\hline \multicolumn{3}{|l|}{ Age (years) } \\
\hline$<25$ & 314 & 12.64 \\
\hline $25-29$ & 1274 & 51.27 \\
\hline $30-34$ & 638 & 25.67 \\
\hline$\geq 35$ & 259 & 10.42 \\
\hline \multicolumn{3}{|l|}{ Residential region } \\
\hline East & 936 & 37.67 \\
\hline Central & 770 & 30.99 \\
\hline West & 779 & 31.35 \\
\hline \multicolumn{3}{|l|}{ Ethnicity } \\
\hline Han & 2345 & 94.37 \\
\hline Minority & 140 & 5.63 \\
\hline \multicolumn{3}{|l|}{ Educational level } \\
\hline High school or below & 700 & 28.17 \\
\hline College or above & 1785 & 71.83 \\
\hline \multicolumn{3}{|l|}{ Annual household income (RMB Yuan) } \\
\hline Low income $(<80,000)$ & 553 & 22.25 \\
\hline Lower medium income $(80,000-109,999)$ & 679 & 27.32 \\
\hline Higher medium income $(110,000-199,999)$ & 485 & 19.52 \\
\hline High income $(>200,000)$ & 768 & 30.91 \\
\hline \multicolumn{3}{|l|}{ Occupation } \\
\hline Unemployed & 592 & 23.82 \\
\hline Manual occupation & 1375 & 55.33 \\
\hline Non-manual occupation & 518 & 20.85 \\
\hline \multicolumn{3}{|l|}{ Pregnancy characteristics } \\
\hline \multicolumn{3}{|l|}{ Parity } \\
\hline Nulliparity & 1517 & 61.05 \\
\hline Multiparity & 968 & 38.95 \\
\hline \multicolumn{3}{|l|}{ Pregnancy plan } \\
\hline Planned & 1849 & 74.41 \\
\hline Unintended & 636 & 25.59 \\
\hline \multicolumn{3}{|l|}{ Health characteristics } \\
\hline \multicolumn{3}{|l|}{ Prepregnancy BMI (kg/m2) } \\
\hline$<18.5$ & 325 & 13.08 \\
\hline $18.5 \square 23.9$ & 1595 & 64.19 \\
\hline$\geq 24$ & 565 & 22.74 \\
\hline \multicolumn{3}{|l|}{ History of smoking } \\
\hline No & 2424 & 97.55 \\
\hline Yes & 61 & 2.45 \\
\hline \multicolumn{3}{|l|}{ History of drinking } \\
\hline No & 2356 & 94.81 \\
\hline Yes & 129 & 5.19 \\
\hline
\end{tabular}

Page 7/12 
Table 2. Comparison of PA between early and mid-to-late pregnancy among Chinese pregnant women $(n=2485)$

\begin{tabular}{|c|c|c|c|c|c|}
\hline \multirow[t]{2}{*}{ PA indicators } & \multicolumn{2}{|l|}{ Early pregnancy } & \multicolumn{2}{|c|}{ Mid-to-late pregnancy } & \multirow[t]{2}{*}{$P$ value } \\
\hline & Mean (SD) & Median (IQR) & Mean (SD) & Median (IQR) & \\
\hline TEE on PA (MET min/week) & 791.93 (1057.56) & $396.00(66.00,1152.00)$ & $1193.14(1283.51)$ & $813.00(356.40,1411.00)$ & $<0.001$ \\
\hline \multicolumn{6}{|l|}{ High-intensity PA } \\
\hline Energy expenditure (MET min/week) & $43.24(327.85)$ & $0.00(0.00,0.00)$ & $86.16(506.34)$ & $0.00(0.00,0.00)$ & $<0.001$ \\
\hline Proportion due to TEE on PA (\%) & $2.46(11.79)$ & $0.00(0.00,0.00)$ & $2.96(12.54)$ & $0.00(0.00,0.00)$ & $<0.001$ \\
\hline \multicolumn{6}{|l|}{ Medium-intensity PA } \\
\hline Energy expenditure (MET min/week) & $73.02(316.18)$ & $0.00(0.00,0.00)$ & $124.45(429.27)$ & $0.00(0.00,0.00)$ & $<0.001$ \\
\hline Proportion due to TEE on PA (\%) & $5.39(16.04)$ & $0.00(0.00,0.00)$ & $7.03(17.09)$ & $0.00(0.00,0.00)$ & $<0.001$ \\
\hline \multicolumn{6}{|l|}{ Walking } \\
\hline Energy expenditure (MET min/week) & $675.66(862.19)$ & $396.00(49.50,990,00)$ & $982.53(929.47)$ & $693.00(297.00,1386.00)$ & $<0.001$ \\
\hline Proportion due to TEE on PA (\%) & $92.15(20.83)$ & $100.00(100.00,100.00)$ & $90.00(22.06)$ & $100.00(100.00,100.00)$ & $<0.001$ \\
\hline
\end{tabular}

Note: The Wilcoxon signed-rank test was used to compare PA indic ators between early and mid-to-late pregnancy. A Pvalue $<0.05$ was considered significant.

Table 3. Associations between population characteristics and sufficient PA in mid-to-late pregnancy 


\begin{tabular}{|c|c|c|c|c|c|}
\hline \multirow[t]{2}{*}{ Characteristics } & \multicolumn{5}{|c|}{ Sufficient PA in mid-to-late pregnancy $(n=1373)$} \\
\hline & $\mathrm{N}(\%)$ & Unadjusted OR (95\%CI) & $P$ value & Adjusted OR (95\%CI) & $P$ value \\
\hline \multicolumn{6}{|l|}{ Demographic characteristics } \\
\hline \multicolumn{6}{|l|}{ Age (years) } \\
\hline$<25$ & $158(11.51)$ & 1.000 & & 1.000 & \\
\hline $25-29$ & $730(53.17)$ & $1.325(1.034,1.697)$ & 0.026 & $1.212(0.922,1.592)$ & 0.168 \\
\hline $30-34$ & $342(24.91)$ & $1.141(0.870,1.495)$ & 0.340 & $1.068(0.783,1.457)$ & 0.677 \\
\hline$\geq 35$ & $143(10.42)$ & $1.217(0.875,1.693)$ & 0.243 & $1.186(0.808,1.743)$ & 0.384 \\
\hline \multicolumn{6}{|l|}{ Region } \\
\hline East & 527 (38.38) & 1.000 & & 1.000 & \\
\hline Central & $368(26.80)$ & $0.710(0.587,0.860)$ & 0.001 & $0.747(0.609,0.916)$ & 0.005 \\
\hline West & $478(34.81)$ & $1.232(1.016,1.496)$ & 0.034 & $1.247(1.012,1.537)$ & 0.038 \\
\hline \multicolumn{6}{|l|}{ Ethnicity } \\
\hline Han & $1296(94.39)$ & 1.000 & & 1.000 & \\
\hline Minority & $77(5.61)$ & $0.989(0.702,1.394)$ & 0.951 & $0.976(0.677,1.408)$ & 0.898 \\
\hline \multicolumn{6}{|l|}{ Educational level } \\
\hline High school or below & $371(27.02)$ & 1.000 & & 1.000 & \\
\hline College or above & $1002(72.98)$ & $1.135(0.952,1.352)$ & 0.158 & $1.023(0.818,1.279)$ & 0.842 \\
\hline \multicolumn{6}{|l|}{ Annual household income (RMB Yuan) } \\
\hline Low inc ome $(<80,000)$ & $295(21.49)$ & 1.000 & & 1.000 & \\
\hline Lower medium income $(80,000-109,999)$ & 359 (26.15) & $0.981(0.783,1.229)$ & 0.868 & $0.950(0.745,1.211)$ & 0.680 \\
\hline Higher medium income $(110,000-199,999)$ & $288(20.98)$ & $1.279(0.999,1.636)$ & 0.051 & $1.185(0.901,1.557)$ & 0.224 \\
\hline High income $(>200,000)$ & 431 (31.39) & $1.119(0.898,1.393)$ & 0.318 & $1.096(0.853,1.409)$ & 0.472 \\
\hline \multicolumn{6}{|l|}{ Occupation } \\
\hline Unemployed & $316(23.02)$ & 1.000 & & 1.000 & \\
\hline Manual occupation & $756(55.06)$ & $1.067(0.879,1.294)$ & 0.513 & $0.888(0.702,1.124)$ & 0.324 \\
\hline Non-manual occupation & $301(21.92)$ & $1.212(0.955,1.537)$ & 0.114 & $1.089(0.838,1.415)$ & 0.525 \\
\hline \multicolumn{6}{|l|}{ Pregnancy characteristics } \\
\hline \multicolumn{6}{|l|}{ Parity } \\
\hline Nulliparity & $856(62,35)$ & 1.000 & & 1.000 & \\
\hline Multiparity & 517 (37.65) & $0.882(0.746,1.043)$ & 0.141 & $0.926(0.759,1.131)$ & 0.452 \\
\hline \multicolumn{6}{|l|}{ Pregnancy planning } \\
\hline Planned & $1038(75.60)$ & 1.000 & & 1.000 & \\
\hline Unintended & $335(24.40)$ & $0.870(0.726,1.042)$ & 0.130 & $0.938(0.773,1.139)$ & 0.520 \\
\hline \multicolumn{6}{|l|}{ Health characteristics } \\
\hline \multicolumn{6}{|l|}{ Prepregnancy BMI (kg/m2) } \\
\hline$<18.5$ & $183(13.33)$ & $1.039(0.817,1.321)$ & & $1.069(0.829,1.377)$ & \\
\hline $18.5 \square 23.9$ & $883(64.31)$ & 1.000 & 0.755 & 1.000 & 0.608 \\
\hline$\geq 24$ & 307 (22.36) & $0.959(0.791,1.163)$ & 0.674 & $1.035(0.842,1.272)$ & 0.746 \\
\hline \multicolumn{6}{|l|}{ History of smoking } \\
\hline No & 1349 (98.25) & 1.000 & & 1.000 & \\
\hline Yes & $24(1.75)$ & $0.517(0.307,0.870)$ & 0.013 & $0.551(0.315,0.964)$ & 0.037 \\
\hline \multicolumn{6}{|l|}{ History of drinking } \\
\hline No & $1304(94.97)$ & 1.000 & & 1.000 & \\
\hline Yes & $69(5.03)$ & $0.928(0.650,1.323)$ & 0.678 & $1.046(0.714,1.531)$ & 0.819 \\
\hline
\end{tabular}

Page 9/12 


\begin{tabular}{|c|c|c|c|c|c|}
\hline Insufficient & 836 (60.89) & 1.000 & & 1.000 & \\
\hline Sufficient & 537 (39.11) & $1.946(1.635,2.315)$ & $<0.001$ & $1.897(1.583,2.274)$ & $<0.001$ \\
\hline
\end{tabular}

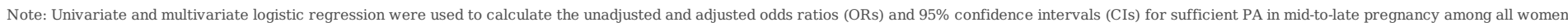
included in the study. A $P$ value $<0.05$ was considered significant, and significant values are marked with bold text.

Table 4. Associations between population characteristics and increasing or decreasing PA across pregnancy 


\begin{tabular}{|c|c|c|c|c|c|c|c|c|c|c|}
\hline \multirow[t]{3}{*}{ Characteristics } & \multicolumn{5}{|c|}{ Increasing PA (insufficient PA in early pregnancy and sufficient PA in mid-to-late } & \multicolumn{5}{|c|}{ Decreasing PA (sufficient PA in early pregnancy and insufficient PA in mid-to-late } \\
\hline & \multicolumn{5}{|c|}{ pregnancy) $(\mathrm{n}=836)$} & \multicolumn{5}{|c|}{ pregnancy) $(n=276)$} \\
\hline & n $(\%)$ & Unadjusted OR $(95 \% \mathrm{CI})$ & $P$ value & Adjusted OR (95\% CI) & Pvalue & $\mathrm{n}(\%)$ & Unadjusted OR $(95 \% \mathrm{CI})$ & Pvalue & Adjusted OR (95\% CI) & $P$ value \\
\hline \multicolumn{11}{|l|}{ Region } \\
\hline East & 309 (36.96) & 1.000 & & 1.000 & & $100(36.23)$ & 1.000 & & 1.000 & \\
\hline Central & $215(25.72)$ & $0.698(0.552,0.883)$ & 0.003 & $0.721(0.562,0.925)$ & 0.010 & $94(34.06)$ & $1.339(0.944,1.899)$ & 0.101 & $1.274(0.880,1.844)$ & 0.199 \\
\hline West & $312(37.32)$ & $1.425(1.128,1.880)$ & 0.003 & $1.387(1.078,1.783)$ & 0.011 & $82(29.71)$ & $1.077(0.755,1.536)$ & 0.683 & $1.048(0.716,1.532)$ & 0.811 \\
\hline \multicolumn{11}{|l|}{ Age (years) } \\
\hline$<25$ & $103(12.32)$ & 1.000 & & 1.000 & & $34(12.32)$ & 1.000 & & 1.000 & \\
\hline $25-29$ & $442(52.87)$ & $1.292(0.962,1.735)$ & 0.089 & $1.251(0.904,1.731)$ & 0.178 & 139 (50.36) & $0.781(0.486,1.253)$ & 0.781 & $0.911(0.542,1.530)$ & 0.724 \\
\hline $30-34$ & $204(24.40)$ & $1.108(1.801,1.533)$ & 0.536 & $1.105(0.763,1.601)$ & 0.598 & $78(28.26)$ & $0.914(0.549,1.522)$ & 0.914 & $1.103(0.615,1.978)$ & 0.742 \\
\hline$\geq 35$ & $87(10.41)$ & $1.132(0.763,1.678)$ & 0.537 & $1.107(0.699,1.754)$ & 0.664 & $25(9.06)$ & $0.722(0.382,1.365)$ & 0.722 & $0.775(0.375,1.601)$ & 0.491 \\
\hline \multicolumn{11}{|l|}{ Ethnicity } \\
\hline Han & $788(94.26)$ & 1.000 & & 1.000 & & 259 (93.84) & 1.000 & & 1.000 & \\
\hline Minority & $48(5.74)$ & $1.046(0.690,1.586)$ & 0.832 & $0.944(0.606,1.471)$ & 0.799 & $17(6.16)$ & $1.150(0.620,2.131)$ & 0.658 & $1.084(0.558,2.106)$ & 0.811 \\
\hline \multicolumn{11}{|l|}{ Educational level } \\
\hline Senior school or below & 244 (29.19) & 1.000 & & 1.000 & & $84(30.43)$ & 1.000 & & 1.000 & \\
\hline Undergraduate or above & $592(70.81)$ & $1.006(0.815,1.242)$ & 0.957 & $0.879(0.671,1.150)$ & 0.348 & $192(69.57)$ & $0.708(0.512,0.979)$ & 0.037 & $0.662(0.442,0.991)$ & 0.045 \\
\hline \multicolumn{11}{|l|}{ Annual household income (RMB Yuan) } \\
\hline Low income $(<80,000)$ & $183(21.89)$ & 1.000 & & 1.000 & & $62(22.46)$ & 1.000 & & 1.000 & \\
\hline Lower medium income $(80,000-$ & $226(27.03)$ & $1.013(0.772,1.329)$ & 0.927 & $1.017(0.759,1.363)$ & 0.910 & $81(29.35)$ & $1.100(0.726,1.667)$ & 0.652 & $1.262(0.806,1.978)$ & 0.305 \\
\hline \multicolumn{11}{|l|}{$109,999)$} \\
\hline Higher medium inc ome $(110,000-$ & $178(21.29)$ & $1.343(0.996,1.810)$ & 0.053 & $1.345(0.964,1.875)$ & 0.081 & $55(19.93)$ & $0.903(0.577,1.414)$ & 0.656 & $1.086(0.669,1.763)$ & 0.740 \\
\hline \multicolumn{11}{|l|}{ 199,999) } \\
\hline High income $(>200,000)$ & $249(29.78)$ & $1.030(0.789,1.344)$ & 0.830 & $1.087(0.801,1.474)$ & 0.593 & $78(28.26)$ & $0.774(0.515,1.164)$ & 0.219 & $0.896(0.565,1.419)$ & 0.639 \\
\hline \multicolumn{11}{|l|}{ Occupation } \\
\hline Unemployed & $206(24.64)$ & 1.000 & & 1.000 & & 59 (21.38) & 1.000 & & 1.000 & \\
\hline Manual occupation & 446 (53.35) & $1.037(0.823,1.307)$ & 0.757 & $0.926(0.696,1.232)$ & 0.597 & $166(60.14)$ & $0.998(0.691,1.443)$ & 0.993 & $1.221(0.795,1.876)$ & 0.362 \\
\hline Non-manual occupation & $184(22.01)$ & $1.168(0.879,1.550)$ & 0.284 & $1.104(0.809,1.505)$ & 0.533 & 51 (18.48) & $0.813(0.515,1.283)$ & 0.373 & $0.978(0.592,1.616)$ & 0.931 \\
\hline \multicolumn{11}{|l|}{ Pregnancy characteristics } \\
\hline \multicolumn{11}{|l|}{ Parity } \\
\hline Nulliparity & $527(63.04)$ & 1.000 & & 1.000 & & $168(60.87)$ & 1.000 & & 1.000 & \\
\hline Multiparity & 309 (36.96) & $0.847(0.692,1.038)$ & 0.110 & $0.881(0.694,1.118)$ & 0.297 & $108(39.13)$ & $1.039(0.766,1.411)$ & 0.805 & $0.888(0.613,1.288)$ & 0.532 \\
\hline \multicolumn{11}{|l|}{ Pregnancy planning } \\
\hline Planned & $629(75.24)$ & 1.000 & & 1.000 & & 197 (71.38) & 1.000 & & 1.000 & \\
\hline Unintended & 207 (24.76) & $0.910(0.731,1.134)$ & 0.401 & $0.988(0.781,1.250)$ & 0.921 & 79 (28.62) & $1.281(0.923,1.779)$ & 0.138 & $1.218(0.860,1.725)$ & 0.266 \\
\hline \multicolumn{11}{|l|}{ Health characteristics } \\
\hline \multicolumn{11}{|l|}{ Pre-pregnancy BMI (kg/m2) } \\
\hline$<18.5$ & $103(12.32)$ & $0.924(0.689,1.240)$ & 0.600 & $0.953(0.701,1.297)$ & 0.761 & $31(11.23)$ & $0.751(0.478,1.181)$ & 0.215 & $1.709(0.439,1.143)$ & 0.158 \\
\hline $18.5 \llbracket 23.9$ & $534(63.88)$ & 1.000 & & 1.000 & & $180(65.22)$ & 1.000 & & 1.000 & \\
\hline$\geq 24$ & $199(23.80)$ & $1.027(0.815,1.295)$ & 0.820 & $1.123(0.877,1.437)$ & 0.359 & $65(23.55)$ & $1.167(0.817,1.667)$ & 0.396 & $1.152(0.787,1.687)$ & 0.467 \\
\hline \multicolumn{11}{|l|}{ History of smoking } \\
\hline No & $821(98.21)$ & 1.000 & & 1.000 & & $269(97.46)$ & 1.000 & & 1.000 & \\
\hline Yes & 15 (1.79) & $0.491(0.262,0.919)$ & 0.026 & $0.480(0.242,0.955)$ & 0.036 & $7(2.54)$ & $1.527(0.562,4.144)$ & 0.406 & $1.446(0.515,4.061)$ & 0.484 \\
\hline
\end{tabular}




\section{Figures}

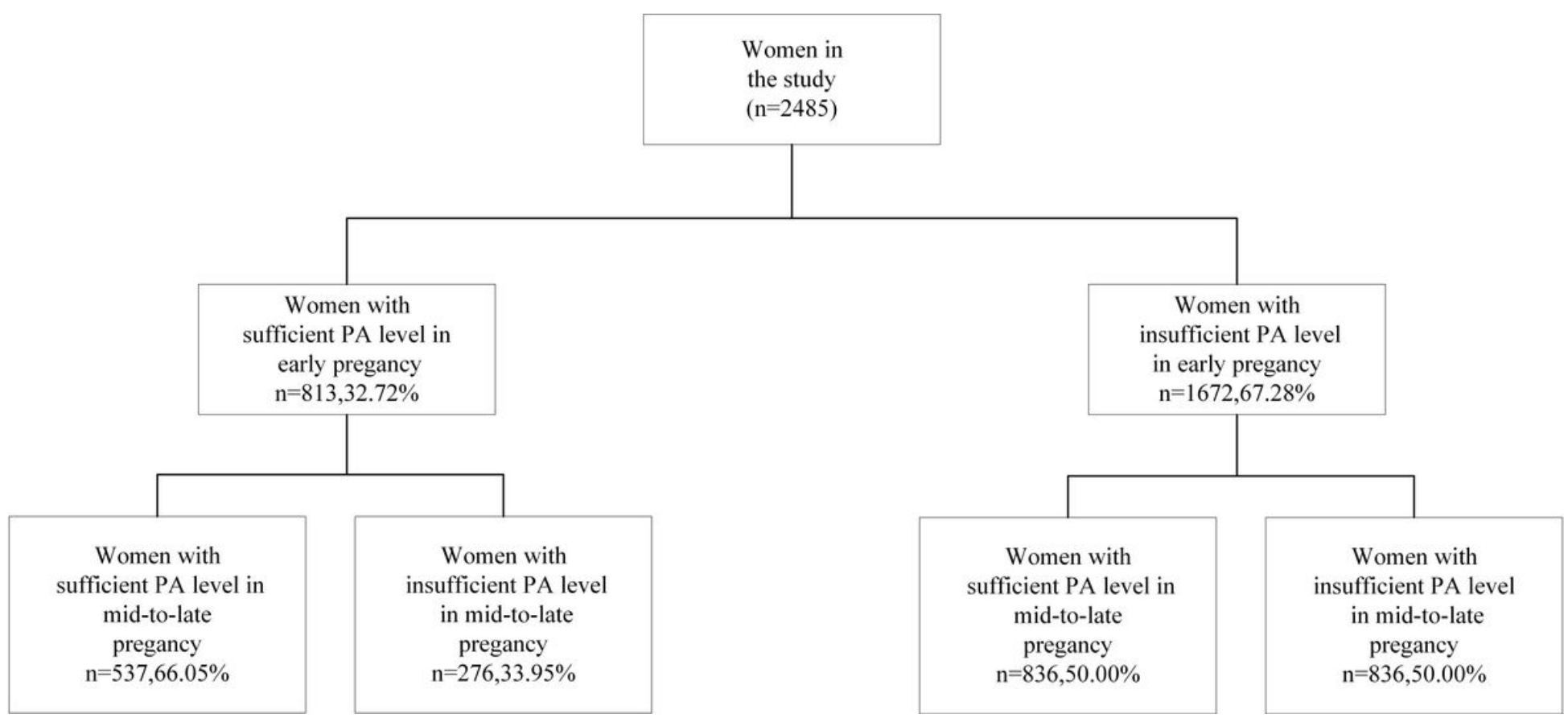

Figure 1

The proportions of women with sufficient and insufficient PA levels in early and mid-to-late pregnancy 